\title{
Demand-Aware Price Policy Synthesis and Verification Services for Smart Grids
}

\author{
Toni Mancini, Federico Mari, Igor Melatti, \\ Ivano Salvo, and Enrico Tronci \\ Sapienza University of Rome, Italy
}

\author{
Jorn Klaas Gruber, Barry Hayes, \\ and Milan Prodanovic \\ Instituto IMDEA Energía, Spain
}

\author{
Lars Elmegaard \\ SEAS-NVE, Denmark
}

\begin{abstract}
In management tasks for modern electricity networks the stakeholders face typically two conflicting objectives: maximization of income (increasing demand) and reduction of demand peaks (reducing costs). To improve management of electricity distribution networks, an integrated service-based methodology is presented in this paper. Namely, the proposed approach: i) computes the operational constraints in order to improve utilization of the whole network; ii) enforces those constraints by focusing on each network substation separately; iii) verifies that probability of violating those constraints in nonnominal cases is fairly low. The feasibility of the approach has been tested tested by using a realistic scenario taken from an existing medium voltage Danish distribution network. In such scenario, the proposed method improves the network utilization and offers economic benefits for all the principal participants, i.e. DSOs, retailers and end users.
\end{abstract}

\section{INTRODUCTION}

One of the most challenging problems in modern electricity smart grids management is to find a trade-off between two conflicting goals. Namely, on one hand both the energy retailer, who sells energy, and Distribution System Operator (DSO), who manages all aspects of the Electric Distribution Network (EDN), want to sell as much energy as possible, without forcing residential end users to cut their power demand. On the other hand, if all users require energy at the same time (peak hour), this may result in an economical damage (both for usage of peak power plants and for transformer loss-of-life [1]) for the DSO.

\section{A. Contribution}

In this paper we introduce a novel methodology to improve EDN management and counteract the problem outlined above (see Fig. 1). Namely, our methodology is based on three integrated services, and exploits the EDN hierarchy induced by EDN substations interconnection. The first service, which we call EDN Virtual Tomography (EVT) service, considers the whole EDN, detects possible violations of network constraints, and computes operational constraints on several EDN components, in order to improve EDN usage. Such constraints may be easily casted by the DSO as desired power profiles on each EDN substation $s$, thus defining the safety conditions for each $s$. In order to enforce substations safety, without limiting the power required by end users, the other two services of our approach go a step down on the EDN hierarchy and work on single substations. Namely the second service, which we call Demand-Aware Price Policy (DAPP) service, redistribute the power demand (load shifting) so that the constraints on the input substation $s$ are fulfilled. This is done by computing,

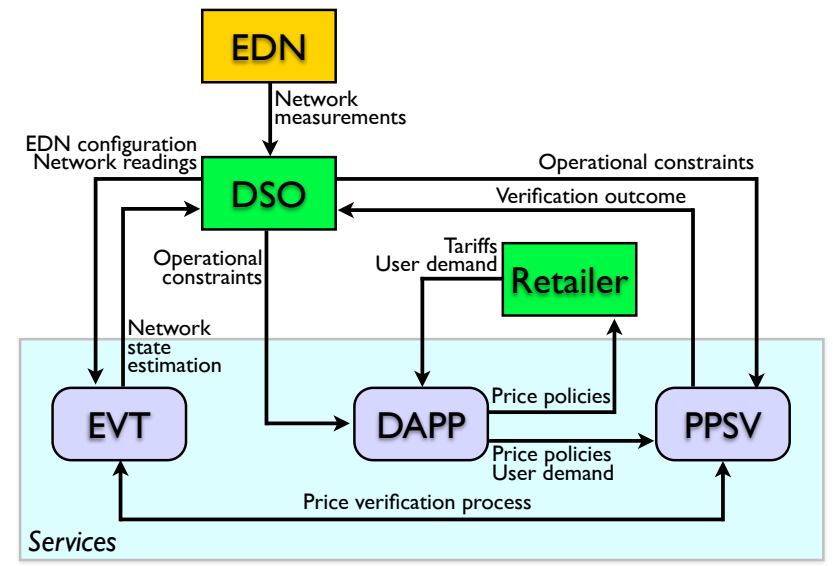

Fig. 1: The proposed services architecture.

for each residential home $u$ connected to $s$, an individualized suggested power profile $P_{u}$ (that is, different users may get different power profiles), so that if all users follow their power profile then the operational constraints on $s$ are met. Each user $u$ is motivated to follow the suggested power profile $P_{u}$ by an individualized price policy based on $P_{u}$. Proposing individualized price policies avoids that power demand peaks are simply shifted through the day (power demand rebounds), which would again violate constraints on substations. Since users are not forced to follow their suggested power profiles, the Price Policy Safety Verification (PPSV) service investigates the consequences of users deviations from power profiles. Namely, given a probabilistic model for such deviations, PPSV is able to compute the probability distribution of the resulting aggregated power demand. Such probability distribution is used to feed back EVT, in order to simulate the effect of the price policies on the EDN. Finally, such simulation results are evaluated and the final verification outcome is sent to the DSO. More in detail, our services may be described as follows.

1) EVT: The EVT service uses measurements of the local energy consumption and generation obtained at certain points in the EDN to compute voltages, currents and other physical values for locations in the EDN not directly accessible by sensors. The EVT service is intended to help the DSO to improve EDN operating conditions, through improved network observability, even in parts of the EDN where sensors are not available. In addition, the EVT service is used to check the technical feasibility of the price policies generated by the PPSV service (see Fig. 1). 
2) DAPP: The main goal of DAPP is to optimize EDN operation at substation level, by avoiding peaks (peak shaving). In order to do this, DAPP individually motivates users to perform load shifting. This is achieved by proposing individualized price policies to each of the homes connected to a given EDN substation. Namely, the price policy output by DAPP for user $u$ (also called DAPP tariff in the following) is based on a power profile region defining a low tariff area. Namely, when user demand is inside [outside] the low tariff area, the user will pay a low [high] tariff (high and low tariffs are decided by the energy retailer). Note that, in order for DAPP to propose economically viable individualized price policies, it must be easy for each user to keep the demand inside the low tariff area most of the time, thus lowering down the energy bill.

3) PPSV: The main goal of PPSV is to verify safety of a given substation $s$, when to each residential user connected to $s$ is applied the corresponding individualized price policy computed by the DAPP service. Since not all users will be able to exactly follow their proposed price policies, PPSV requires as input a probabilistic disturbance model describing how residential users are foreseen to deviate. Such model may be computed from historical data on user demand. As a result, PPSV returns the probability distribution of the aggregated power demand on $s$ (i.e., the probability that the aggregated power demand is in a given interval).

This paper is organized as follows. Sects. II, III and IV describe the algorithms behind each service in our approach (resp., EVT, DAPP and PPSV). Finally, Section V describes a meaningful and real-world scenario, on which our approach is evaluated. Such an evaluation is performed on both a technical and an economical perspective, in order to show that our approach is economically viable for both DSOs (as it improves EDN management) and residential users (as it lowers down their energy bills).

\section{B. Related Work}

Distribution systems are designed to deliver power from the bulk transmission grid to end-users. Traditionally, power flowed in one direction through the EDNs along radial feeders, and these power flows were relatively straightforward to predict based on historical data. With the large-scale integration of Distributed Energy Resources (DER), such as Photovoltaic (PV) installations, demand-responsive loads, and storage devices, power flows in the EDN become much more complex and variable. As a consequence of this, more detailed knowledge of the EDN state is required, and a more active approach to managing network congestions and constraints is required from DSOs. This has led to the adoption of network management techniques in EDNs, which were previously only used in transmission systems, such as state estimation and advanced energy management systems [2]. State estimation has been a standard feature of transmission network operation for several decades, where it is applied to improve the observability of the network, and reduce the impacts of noise and errors in system measurement data [3]. Recently, there has been significant research interest in developing state estimators specifically for use in EDNs [4]. Distribution network state estimation is often applied as part of an advanced distribution management system, designed to monitor and optimise the energy flows and operation of active EDNs with significant DER [5], [6]. The EVT service developed in this paper employs state estimation to the EDN in order to accurately determine the grid state, and then uses this information to provide warnings, alarms and recommendations to the DSO.

Demand Side Management (DSM) [7] approaches are used to regulate EDN by acting, directly or indirectly, on end users. There are two main approaches to DSM, namely Direct Load Control (DLC) and Autonomous Demand Response (DR). With a DLC approach the utility (DSO) remotely controls energy consumption by curtailing high-load household appliances. With a DR approach each user receives an energy price policy for the next few days. Users will autonomously manage their loads (e.g., by reducing their consumption at peak hours) and their generation (say, from PV panels) so as to minimize their energy bill. This, in turn, will optimize EDN operations. DAPP follows a DR approach, based on the Inclining Block Rate (e.g., see [8]) approach. Note that indeed DAPP synthesizes price policies starting from system level formal specifications. Other works on synthesis from system level formal specifications (focusing on synthesis of controllers for hybrid systems) are in [9], [11], [12], [10]. As for price policies computation, many works are based on distributed algorithms and architectures (e.g., as in [13]). On the contrary, DAPP is based on a centralized algorithm which computes all required price policies. A centralized (Neural Network based) approach similar to the one in DAPP is in [14]. Differently from [14], DAPP assigns a different price policy to each user in the same area (e.g., connected to the same substation), considers the case where users are producing energy, and uses a Linear Programming (LP) based approach. Finally, we point out that the mechanism used by DAPP to encourage users to follow such a suggested behaviour is pricing, which is decided by the energy retailer based on economical as well as social considerations, as described, e.g., in [15].

In statistical model checking (e.g., see [16], [17], [18], [19]) approaches, scenarios are sampled until a certain degree of confidence in the estimated distribution probability (of safety violation) is achieved. PPSV is based on such techniques, and adapts the works in [18], [19] to the smart grid context. In a smart grid context, usage of exhaustive methods (instead of statistical ones) for safety analysis purposes has been studied, e.g., in [20]. However they are plagued by the so called state explosion problem. Since PPSV aims at evaluating safety of a large system (hundreds of homes connected to a substation), the approaches used in the above papers cannot be used in our context. An exhaustive simulation-based approach (applied on verification of satellite procedures) similar to the one used for PPSV has also been studied in [21].

Summing up, to the best of our knowledge, the integrated service-based approach we present here has not been previously proposed in the literature.

\section{EDN VIRTUAL TOMOGRAPHY}

The supervision and control of distribution networks becomes increasingly important with the integration of Distributed Energy Resources (DER). High penetrations of DER results in an increase in the variability of EDN power flows and, as a direct consequence, more detailed knowledge on the network state is required in order to manage the EDN. However, in most EDNs only few measurements are taken due to technical or economic issues. The use of a detailed simulation 


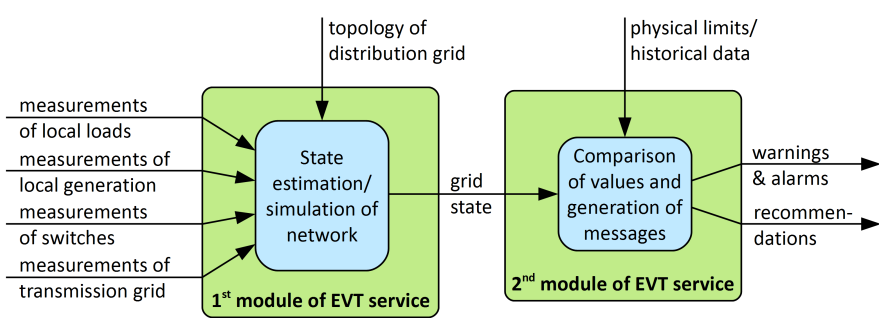

Fig. 2: Inputs and outputs of the two modules of EVT.

model of an EDN allows a wide range of physical values to be computed and the state of the network to be estimated. The results of the state estimation can then be used to automatically generate warnings and alarms if a value approaches or exceeds its limits. The EVT service described below is comprised of two modules, or functions: (A) the estimation of the EDN state; and (B) the generation of warnings, alarms and advice for the DSO.

1) State Estimation: The EVT module to estimate the state of the grid is given in Fig. 2. The inputs are the static network parameters (bus and branch information), along with measurement data (e.g. real-time recordings of voltages, power angles, active/reactive power injections, active/reactive power flows). These data are fed to the state estimator described in [22], which identifies bad data, such as erroneous or missing values in the input measurements. Power flow analysis is then carried out in order to calculate the grid state, expressed as a set of estimates of the voltages, power injections, and power flows throughout the EDN.

2) EVT Warnings, Alarms and Recommendations: The second function of the EVT uses the estimated grid state to generate warnings and alarms, and give recommendations to the DSO, Fig. 2. This EVT module requires additional information on the physical limits of the EDN, as well as historical data in order to detect unusual values. The EDN physical limits do not frequently change and are shown in Fig. 2 as parameters rather than input values. However, these parameters can be adjusted to allow the EVT to investigate EDN performance during scheduled outages (e.g. lines down due to maintenance), or unscheduled outages (network faults).

The EVT compares the input values and the supplied parameters and checks the limits of different components of the EDN. If limits are violated, alarms are provided, and appropriate corrective actions (e.g. network switching, load management) can be simulated, allowing recommendations to be made. In addition, the EVT can be configured to provide general recommendations to the DSO for managing voltage, congestions and losses in the EDN. This may be casted by the DSO as desired power profiles for EDN substations, which are then used by DAPP and PPSV services.

\section{DEMAND-Aware Price Policy}

In this section we describe our DAPP algorithm. To this aim, we first define the notation we use (which is in common with PPSV). A time-slots set $T$ is a finite set of contiguous time-slots, all having the same duration. A power profile is a function $P: T \rightarrow \mathbb{R}$. A power profile tube (or region) is a pair of power profiles $\left(P_{l}, P_{h}\right)$ defined over the same $T$ s.t. $P_{l}(t) \leq P_{h}(t)$ for all $t \in T$. A power profile $P$ follows a power profile tube $\left(P_{l}, P_{h}\right)$ if and only if $P_{l}(t) \leq P(t) \leq P_{h}(t)$

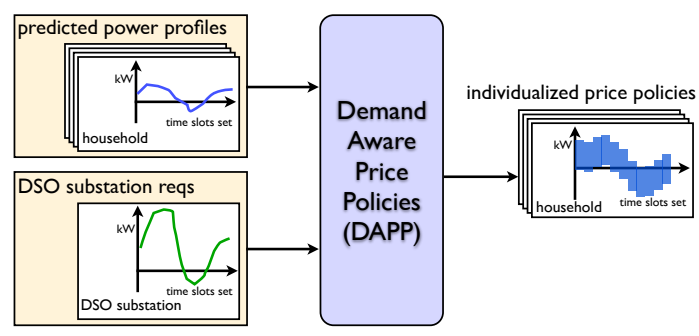

Fig. 3: DAPP input and output on a single DSO substation.

for all $t \in T$. Finally, a Linear Programming (LP) problem is a minimization problem over a set of linear inequalities (constraints) on real variables.

We may now describe in detail input and output for our DAPP algorithm (for a high-level view, see Fig. 3). Namely, DAPP requires the following input: (i) a set of homes $U$ connected to a substation $s$; (ii) a time-slots set $T$ (typically with a time span one month in the future); (iii) the desired power profile $P_{s}$ on $T$ for the substation $s$, as decided by the DSO on the basis of EVT output; (iv) four per-unit tariffs $b_{l} \leq b_{h}, s_{l} \leq s_{h} \in \mathbb{R}^{+}$coming from the energy retailer: respectively, the low buy, high buy, low sell, and high sell tariffs; (v) for each user $u \in U$, a power profile tube $\left(Q_{u, l}, Q_{u, h}\right)$ on $T$, so that the actual power profile for user $u$ is foreseen to follow $\left(Q_{u, l}, Q_{u, h}\right)$ on $T$. For a method to compute such a demand forecasting, see e.g. [23]. Finally, the output of DAPP is a set of individualized price policies, defined on the basis of individualized power profile regions $\left(P_{u, l}, P_{u, h}\right)$ on $T$, which we also refer to as low tariff areas, for each $u \in U$. Namely, the DAPP (output) tariff, for a given user $u$, is defined to incentivize the user to follow the output power profile region as follows: if $u$ follows $\left(P_{u, l}, P_{u, h}\right)$, then either $b_{l}$ (if $u$ is consuming energy) or $s_{h}$ (if $u$ is producing energy) is applied, otherwise $b_{h}$ and $s_{l}$ are applied, respectively.

\section{A. DAPP Algorithm}

In order to achieve such input-output behavior, DAPP creates and solves an LP problem $\mathcal{P}$ defined by the following constraints. Our goal is to provide each user with a proposed power profile $\left(P_{u, l}, P_{u, h}\right)$ s.t. i) the user is incentivated to follow it and ii) if all users follow it, then the aggregated power demand on $s$ is nearly always below $P_{s}$. In order to achieve the first goal, our LP $\mathcal{P}$ states that $\left(P_{u, l}, P_{u, h}\right)$ must be kept as close as possible to users forecasted actual needs $\left(Q_{u, l}, Q_{u, h}\right)$. To this end, $\mathcal{P}$ minimizes the maximum distance between $\left(P_{u, l}, P_{u, h}\right)$ and $\left(Q_{u, l}, Q_{u, h}\right)$. Moreover, $\mathcal{P}$ states that $\left(P_{u, l}, P_{u, h}\right)$ must be wide enough, i.e. $P_{u, h}(t)-P_{u, l}(t) \geq 1$ $\mathrm{kW}$ for all $u \in U, t \in T$. As for the second goal, $\mathcal{P}$ states that the maximum aggregated power demand on a given time-slot $t$ (i.e., $\hat{P}(t)=\sum_{u \in U} P_{u, h}(t)$ ) must be kept lower than the safety bound $P_{s}(t)$. However, if for some $t$ the aggregated power demand is greater than $P_{s}(t)$ (i.e., $\left.\hat{Q}(t)=\sum_{u \in U} Q_{u, h}(t) \geq P_{s}(t)\right)$, this would be unfeasible. Thus, for such $t$, our LP $\mathcal{P}$ states that $\hat{P}(t)$ must be at most the average on $T$ of $\hat{Q}(t)$. In this way, the exceeding power demand is re-distributed among all users in a fair way. Finally, regulations or economic considerations typically limit the maximum amount of energy sent to the grid. To this aim, $\mathcal{P}$ states that the aggregated minimum power must be greater than 0: $\sum_{u \in U} P_{u, l}(t) \geq 0$. Summing up, DAPP algorithm consists 


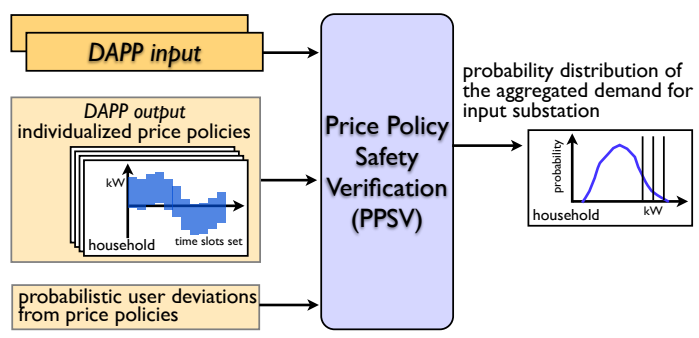

Fig. 4: PPSV input and output on a single DSO substation.

in creating an LP problem $\mathcal{P}$ described as above, solving $\mathcal{P}$ using an LP solver (namely ILOG CPLEX) and finally extracting the actual DAPP output (i.e., values for $\left(P_{u, l}, P_{u, h}\right)$ ) from the solution for $\mathcal{P}$ as returned by the LP solver.

\section{Price Policy SAFETy Verification}

In this section we describe our PPSV algorithm (Fig. 4). PPSV takes as input the following arguments: (i) all inputs required by DAPP (see Sect. III); (ii) the DAPP output $\left(P_{u, l}, P_{u, h}\right)$. From $\left(P_{u, l}, P_{u, h}\right)$ it is possible to compute the $D A P P$ collaborative user, that is, for each residential user $u$, a power profile $\tilde{P}_{u}$ which follows both $u$ (foreseen) power demand tube $\left(Q_{u, l}, Q_{u, h}\right)$ and DAPP proposed low tariff area $\left(P_{u, l}, P_{u, h}\right)$. Computation of the DAPP collaborative user, for each residential user, is performed by suitably adapting the LP problem described in Sect. III; (iii) a quantization step $\gamma \in \mathbb{R}$; (iv) a probabilistic disturbance model describing how users may deviate from their proposed power profile tube. The disturbance model is defined by means of a function dist : $A \rightarrow[0,1]$, being $A$ a finite subset of $[-1,1]$, with $\sum_{d \in A} \operatorname{dist}(d)=1$. Namely, $\operatorname{dist}(d)=p$ iff, for each user $u, p$ is the probability that, in a random time-slot $t$, the actual power used by $u$ in $t$ is $P_{u}^{(d)}(t)=\tilde{P}_{u}(t)(1+d)$; (v) confidence and tolerance on the overall PPSV output $\delta, \varepsilon \in[0,1]$. As for the output, PPSV aims at providing the distribution probability on the aggregated power demand of $s$ induced by the probabilistic disturbance model. In this way, the robustness of DAPP tariffs may be evaluated by checking that the probability that the aggregated power demand is greater than $P_{s}$ (i.e., the probability of safety violation) is low. This must be done for each $v \in P_{s}(T)$, so that the probability of safety violation is checked for each safety bound defined on a time-slot subset. To this aim, PPSV computes a distribution probability aggr $v$ for each $v \in P_{s}(T)$, s.t. with confidence $1-\delta$ the maximum relative error on probabilities is bounded by $\varepsilon$. In formulas, PPSV outputs a set of functions $\left\{\operatorname{aggr}_{v}: R \rightarrow[0,1] \mid v \in P_{s}(T)\right\}$ s.t. $R$ is the finite set of all possible $\gamma$-quantized values (i.e., for all $r_{1} \geq r_{2} \in R, r_{1}-r_{2}=k \gamma$ with $k \in \mathbb{N}$ ) for aggregated disturbed DAPP collaborative profiles $\sum_{u \in U} P_{u}^{(d)}(t)$. Each function $\operatorname{aggr}_{v}$ is s.t. $\operatorname{aggr}_{v}(r)=p$ iff, taken a random timeslot $t \in P_{s}^{-1}(v)$, with confidence $1-\delta$ the probability that $\sum_{u \in U} P_{u}^{(d)}(t) \in[r, r+\gamma)$ is in $[p(1-\varepsilon), p(1+\varepsilon)]$. Finally, pairs $\left(r, \operatorname{aggr}_{v}(r)\right)$ for which $\operatorname{aggr}_{v}(r)>0$ are sent back to the EVT, in order to set meaningful and challenging scenarios for EDN safety verification (see Fig. 1). The final verification outcome computed by EVT is then sent back to the DSO.

\section{A. PPSV Algorithm}

In order to achieve such input-output behavior, for all $v \in P_{s}(T)$ and for all $\gamma$-quantized values $r \in R$ we proceed as follows. Following the Monte-Carlo based approach discussed in [18], [19], we generate $N=f(\delta, \varepsilon)$ random values for the aggregated power demand under the given disturbance scenario. Namely, each of such $N$ values is obtained by picking at random a time-slot $t \in P_{s}^{-1}(v)$ and, for each home $u \in U$, a value $d_{u} \in A$, and then computing $\sum_{u \in U} P_{u}^{\left(d_{u}\right)}(t)$. Finally, $\operatorname{aggr}_{v}(r)=\frac{S}{N}$, being $S$ the number of such trials for which $\sum_{u \in U} P_{u}^{\left(d_{u}\right)}(t) \in[r, r+\gamma)$.

\section{EXPERIMENTAL RESULTS}

In this section we show the technical and economical viability of our approach by presenting the results we obtain on a reference scenario based on the medium voltage EDN actually used in a Danish village and managed by Danish DSO SEAS-NVE. Such EDN is a $10 \mathrm{kV}$ system with a mostly radial structure and a peak demand of $3.2 \mathrm{MW}, 77 \%$ of which is made up of residential users with significant PV generation. Historical hour-by-hour data on residential users energy consumption/production is available from October 2012 to October 2013 (13 months). In order to set up a scenario in which a substation may not be able to satisfy user power demand while retaining its safety, thus making load shifting necessary, we proceed as follows. We choose the substation having the maximum number of homes equipped with heat pumps (which maximizes loads). This results in a substation $s$ with 13 homes. We then create a pool of 130 homes, by replicating 9 times (plus the "original" copy) each of the aforementioned 13 homes. In this way, for some periods of the year (namely, in Winter), the substation is not always able to satisfy all user aggregated power demand while retaining substation safety.

1) EVT Evaluation: Evaluation of EVT is performed in one of the most critical loading scenarios on the given EDN, which take place in February 2013. Here, we present results of EVT when initially invoked to determine network operational constraints (Fig. 5, where price policies have not yet been proposed to users) and when invoked on the most likely worst scenario as computed by the PPSV service (Fig. 6, resulting on applying price policies to end users). In both such figures, MVA flow in each network branch is indicated by the size of the flow arrows (green arrows indicate active power flow, while blue arrows indicate reactive power flow). Larger flow arrows indicate that flow is a higher percentage of the branch MVA thermal rating. The EVT is set up to issue a warning to the DSO when any branch MVA flow exceeds $80 \%$. An alarm is issued if any branch flow exceeds its thermal constraint, i.e. 100\%. In the case of Fig. 5, where DAPP and PPSV are not used, the MVA flow has reached $103 \%$ of its thermal rating, illustrated by the red arrows. As a consequence, EVT advises that the network switches should be re-configured to alter the power flows, or that load management could be carried out in certain parts of the EDN in order to reduce congestion. From such recommendations, the DSO may define the desired power profile for the substation $s$ in the reference scenario to be at $80 \%$ of its nominal power (i.e., $400 \mathrm{kVA}$ ) at certain time periods.

2) DAPP Evaluation: Evaluation of DAPP is performed by running DAPP 13 times from October 2012 to October 

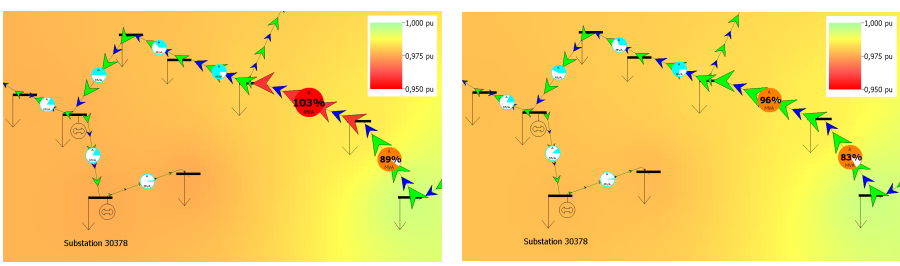

Fig. 5: Detail of EVT output for February 2013, before using DAPP.

Fig. 6: Effect of DAPP on

Fig. 5, using worst scenario computed by PPSV.

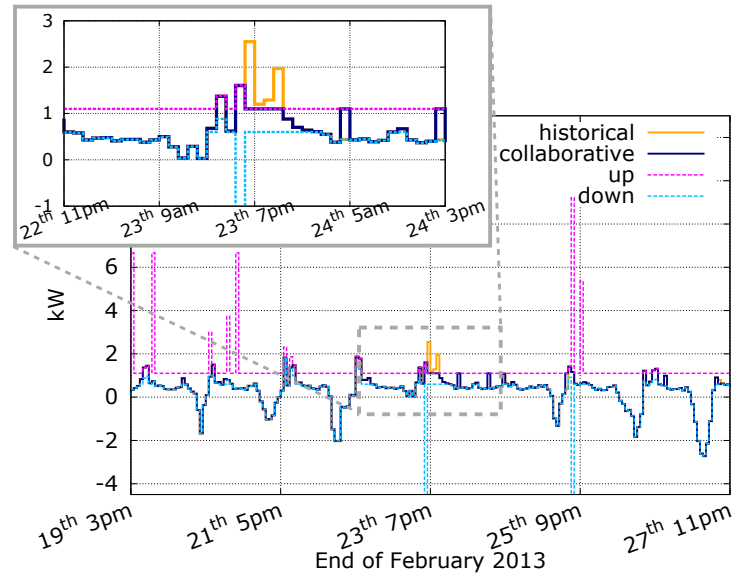

Fig. 7: DAPP output for a household at end of February 2013.

2013 on the reference scenario. As for forecasted power profile region, we define $Q_{u, l}(t)=(1-0.1) \tilde{P}_{u}(t)$ and $Q_{u, h}(t)=(1+0.1) \tilde{P}_{u}(t)$, being $\tilde{P}_{u}(t)$ the users power demand from historical data. This allows us to evaluate DAPP without having to implement a reliable demand forecasting service. As for the energy tariffs, we use the following values: $b_{l}=0.15$, $b_{h}=0.6, s_{l}=s_{h}=0.08 \mathrm{EUR} / \mathrm{kWh}$, which are used in homes involved in a trial run by SEAS-NVE. Finally, as for the desired power profile $P_{s}$, we set it to be always $80 \%$ of substation nominal power (i.e., $P_{s}(t)=320 \mathrm{~kW}$ ). This avoids synchronized peak rebounds among $s$ and other EDN substations, which would be possible if the $80 \%$ limit is set only at peak hours as suggested by EVT output. From the technical perspective, for each month DAPP is able to compute the low tariff areas for all 130 users in the reference scenario in at most 45 minutes, requiring at most $1 \mathrm{~GB}$ of RAM. As a result, if all users follow the DAPP price policies (i.e., they follow the collaborative power profile as computed in Sect. IV), then peaks are indeed avoided, as it is shown in Fig. 8. Namely, Fig. 8 shows both the user power profile from historical data and the DAPP collaborative power profile, both aggregated on all 130 homes in the reference scenario. As a result, while historical data are often even higher of the $120 \%$ of substation nominal profile, collaborative users are never above $100 \%$, and almost always under $80 \%$. This shows that DAPP is indeed able to enforce network safety. From the economical perspective, we compare the two energy bills payed, in the reference scenario, when it is applied (i) the DAPP tariff; (ii) the flat tariff currently used in the village of the reference scenario $(0.28 \mathrm{EUR} / \mathrm{kWh}$ for energy consumption, $0.08 \mathrm{EUR} / \mathrm{kWh}$ for energy production). As for users behavior, in the flat tariff case we assume they follow the power profile from historical data. As for the DAPP tariff case, we take into account two possibilities, thus making two

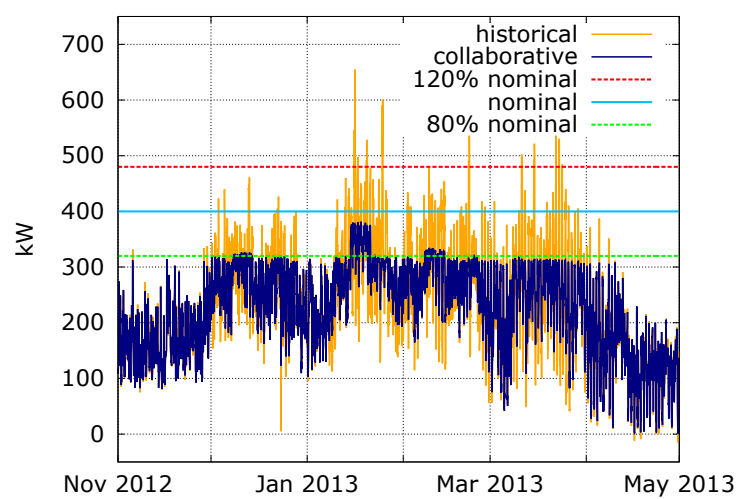

Fig. 8: DAPP output on Winter aggregated on all homes.

different comparisons. In the first one, users ignore the DAPP tariff (i.e., they continue to follow the power profile from historical data). As a result, they obtain, in the reference scenario, a minimum (on the set of 130 homes) $27 \%$ gain $(36 \%$ on average) if the DAPP tariff is applied. This first comparison shows that DAPP tariffs are easy to follow for users. Fig. 7 graphically shows this fact for the period and the home with maximum load by plotting: (i) the DAPP tariff $\left(P_{u, l}, P_{u, h}\right)$, where $P_{u, l}$ is denoted with dashed curve "down" and $P_{u, h}$ with dashed curve "up"; (ii) the DAPP "collaborative" user power profile; (iii) the user power profile from "historical" data (i.e. users which ignore the DAPP tariff). Finally, in the second comparison users with DAPP tariff follow the DAPP collaborative power profile. As a result, the collaborative users obtain a minimum (on the set of 130 homes) $46 \%$ gain $(49 \%$ on average). This second comparison shows that DAPP tariffs enable money saving for end users.

3) PPSV (and back to EVT) Evaluation: Finally, evaluation of PPSV (also showing how EVT handles PPSV output, see Fig. 1) is performed by running PPSV on the reference scenario and using the price policies output by DAPP on the reference scenario. As for the disturbance model, it is defined as follows. We have $A=\{-0.4,-0.2,0,0.2,0.4\}$, thus users may deviate up to $40 \%$. The disturbances distribution probability is designed so that the higher the deviation, the lower the probability. Namely, $\operatorname{dist}( \pm 0.4)=0.1$, dist $( \pm 0.2)=$ 0.2 , dist $(0)=0.4$, thus the probability of a deviation is $60 \%$. Finally, as for the other PPSV input, we set quantization $\gamma=10 \mathrm{~kW}$, tolerance $\epsilon=0.05$ and confidence $1-\delta=99.99 \%$. As a result, from the technical perspective, PPSV completes each of the 13 runs within 13 minutes of computation time at most (7 minutes on average) and 1GB of RAM at most. As for the output evaluation, in Fig. 9 we show the result in the month of February 2013. Fig. 9 may be easily used to show DAPP output robustness on the reference scenario. In fact, by interpreting results from EVT on the whole EDN, we have that the integral of each curve for aggregate demand values beyond $320 \mathrm{~kW}(80 \%$ of the nominal substation power) measures the probability that the substation is overloaded. The integral for aggregate demand values beyond $480 \mathrm{~kW}(120 \%$ of the nominal sustation power) measures the probability that the substation is in unsafe critical conditions. Given this, from Fig. 9 we can observe that, even if users reasonably deviate from their individualized price policies, the probability of a safety violation is very low. Finally, the probability distribution shown in Fig. 9 is sent back to the EVT for the final verification. To this aim, Fig. 6 shows the (selected) output from the EVT 


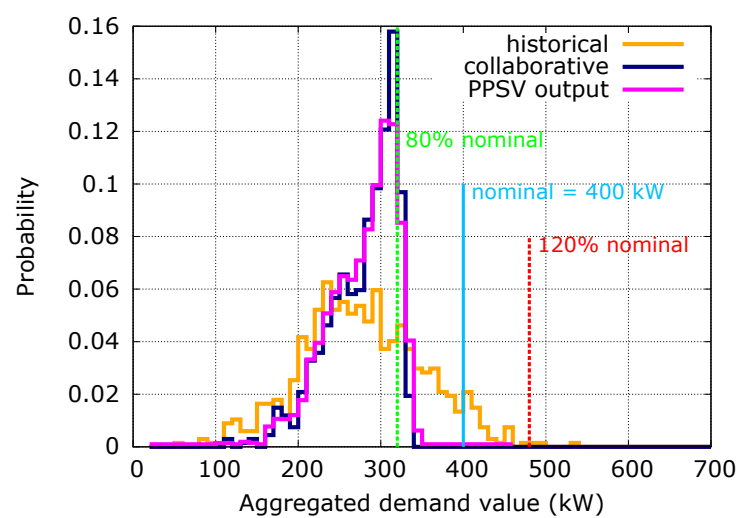

Fig. 9: Probability distributions of the power aggregated demand on February 2013.

for the most likely "worst-case" loading scenario. If compared with Fig. 5 (where the price policies output by DAPP were not applied to end users), we note that the thermal overloading in the EDN has been removed, with the maximum MVA flow at $96 \%$. This price verification process closes the loop between the EVT, DAPP and PPSV services, demonstrating that the application of the three services demonstrated in this paper can be used effectively manage EDN technical constraints.

\section{CONClusions AND Future Work}

In this paper we presented a novel service-based methodology to improve EDN management. Namely, with our approach, all actors involved obtain a gain: residential end users are able to lower down their energy bills, while the energy retailer and the DSO are able to sell as much energy as possible while avoiding EDN overloading (thus preserving EDN safety). Our approach is based on three integrated services: (i) EVT monitors the whole EDN and detect overloaded subnetworks; (ii) given an EDN substation $s$, DAPP proposes individualized price policies (based on individualized power profiles) to all end users connected to the substations, so that desired operational constraints on $s$ are met if each user follows the proposed power profile; (iii) PPSV proves that operational constraints on substations are met with high probability also when users are allowed to (reasonably) deviate from their proposed power profile.

We showed the feasibility of our approach on a medium voltage EDN in a Danish village. Namely, in the reference scenario, EVT was able to suggest to DSO operational constraints on each substation in order to improve EDN usage (by avoiding overloads). By focusing on the most critical of such substations $s$, we showed that DAPP was able to motivate user to move their loads in order to (unknowingly) meet the operational constraints on $s$. Finally, PPSV showed that operational constraints on $s$ in the reference scenario are met even if end users deviate from the proposed power profile for the $60 \%$ of the time.

As future work we plan to further improve our services, by refining methods for dealing with bad data and missing measurements in the input data (EVT), by investigating price policies that explicitly take into account users energy storage capabilities (DAPP) and by devising parallel verification approaches (PPSV).
Acknowledgments: The research leading to these results has received funding from the EU Seventh Framework Programme (FP7/2007-2013) under grant agreement n. 317761 (SmartHG).

\section{REFERENCES}

[1] A. D. Hilshey, P. D. H. Hines, P. Rezaei, and J. R. Dowds, "Estimating the Impact of Electric Vehicle Smart Charging on Distribution Transformer Aging." IEEE Trans. Smart Grid, vol. 4, n. 2, 2013

[2] A. Meliopoulos, E. Polymeneas, Z. Tan, R. Huang, and D. Zhao, "Advanced distribution management system," IEEE Trans. Smart Grid, vol. 4, n. 4, pp. 2109-2117, Dec 2013.

[3] Y.-F. Huang, S. Werner, J. Huang, N. Kashyap, and V. Gupta, "State estimation in electric power grids: Meeting new challenges presented by the requirements of the future grid," Signal Processing Magazine, IEEE, vol. 29, n. 5, pp. 33-43, 2012.

[4] E. Manitsas, R. Singh, B. Pal, and G. Strbac, "Distribution system state estimation using an artificial neural network approach for pseudo measurement modeling," IEEE Trans. Pow. Sys., vol. 27, n. 4, pp. 18881896, Nov 2012.

[5] D. Haughton and G. Heydt, "A linear state estimation formulation for smart distribution systems," IEEE Trans. Pow. Sys., vol. 28, n. 2, pp. 1187-1195, May 2013.

[6] B. Hayes, I. Hernando-Gil, A. Collin, G. Harrison and S. Djokić, "Optimal Power Flow for Maximizing Network Benefits From DemandSide Management," IEEE Trans. Pow. Sys., vol. PP, n. 99, pp. 1-9, 2014.

[7] M. C. Vlot, J. D. Knigge, and J. G. Slootweg, "Economical regulation power through load shifting with smart energy appliances," IEEE Trans. Smart Grid, vol. 4, n. 3, pp. 1705-1712, 2013.

[8] P. Reiss and M. White, "Household electricity demand, revisited," Rev. Econ. Studies, vol. 72, n. 3, pp. 853-883, July 2005.

[9] F. Mari, I. Melatti, I. Salvo, and E. Tronci, "Model based synthesis of control software from system level formal specifications," ACM Trans. SW Eng. and Methodology, vol. 23, n. 1, 2014.

[10] V. Alimguzhin, F. Mari, I. Melatti, I. Salvo, and E. Tronci, "Automatic control software synthesis for quantized discrete time hybrid systems," in Proc. CDC 2012, IEEE, pp. 6120-6125.

[11] - "On-the-fly control software synthesis," in Proc. SPIN 2013, LNCS 7976, Springer

[12] — "A map-reduce parallel approach to automatic synthesis of control software," in Proc. SPIN 2013, LNCS 7976, Springer

[13] Z. Wang and L. Wang, "Adaptive negotiation agent for facilitating bidirectional energy trading between smart building and utility grid," IEEE Trans. Smart Grid, vol. 4, n. 2, pp. 702-710, 2013.

[14] N. Kunwar, Y. K., and R. Kumar, "Area-load based pricing in dsm through ann and heuristic scheduling," IEEE Trans. Smart Grid, vol. 4, n. 3, pp. 1275-1281, 2013.

[15] P. R. Thimmapuram and J. Kim, "Consumers price elasticity of demand modeling with economic effects on electricity markets using an agentbased model," IEEE Trans. Smart Grid, vol. 4, n. 1, pp. 390-397, 2013.

[16] T. Mancini, F. Mari, A. Massini, I. Melatti, F. Merli, and E. Tronci, "System level formal verification via model checking driven simulation," in Proc. CAV 2013, LNCS 8044, Springer

[17] T. Mancini, F. Mari, A. Massini, I. Melatti, and E. Tronci, "System level formal verification via distributed multi-core hardware in the loop simulation," in Proc. PDP 2014, CPS

[18] R. Grosu and S. A. Smolka, "Monte carlo model checking," in Proc. TACAS 2005, LNCS 3440, Springer

[19] P. Dagum, R. M. Karp, M. Luby, and S. M. Ross, "An optimal algorithm for monte carlo estimation," SIAM J. Comput., vol. 29, n. 5, 2000.

[20] M. LeMay and C. Gunter, "Cumulative attestation kernels for embedded systems," IEEE Trans. Smart Grid, vol. 3, n. 2, pp. 744-760, 2012.

[21] G. Verzino, F. Cavaliere, F. Mari, I. Melatti, G. Minei, I. Salvo, Y. Yushtein, and E. Tronci, "Model checking driven simulation of sat procedures," in Proc. SpaceOps 2012.

[22] B. Hayes and M. Prodanović, "Short-term operational planning and state estimation in power distribution networks," in CIRED Workshop 2014.

[23] L. de Castro and P. Cramton, "Prediction markets for electricity demand," in Proc. Allerton 2012. IEEE 